\title{
Narrow Linewidth InGaN Laser Diodes Based on External Cavity Fiber Bragg Grating
}

\author{
Mathilde Gay ${ }^{1}$, Antoine Congar ${ }^{1}$, Dominique Mammez ${ }^{1}$, Laurent Lablonde ${ }^{2}$, Raphaël Butté ${ }^{3}$, Nicolas \\ Grandjean $^{3}$, Pascal Besnard ${ }^{1}$, Stéphane Trebaol $^{1}$ \\ 1. Univ Rennes, CNRS, Institut FOTON - UMR 6082, F-22305 Lannion, France \\ 2. iXblue, rue Paul Sabatier, Lannion, France \\ 3. Institute of Physics, École Polytechnique Fédérale de Lausanne (EPFL), CH-1015 Lausanne, Switzerland
}

The first InGaN-based LDs were reported in 1996 by Nakamura and co-workers [1] and commercialized from 1999 by the Nichia Corporation. Since then, huge efforts have been devoted to the optimization of the epitaxial layers (doping, defect concentration, shortening of the radiative lifetime, etc.). Nowadays, blue laser diodes emitting hundreds of $\mathrm{mW}$ are commercially available but they often exhibit multimode behaviors for both transverse and longitudinal directions. The InGaN-based laser diode technology is still in its infancy, compared to its long wavelength counterparts and only recently the first electrically pumped single mode blue laser diodes have been demonstrated [2] with a resolution limited linewidth of $11.7 \mathrm{GHz}$. At telecom wavelengths, a mature approach to force the laser diode in a single frequency regime consists in using an external feedback by means of Fiber Bragg Grating [3,4]. Nevertheless, to our knowledge, such a compact design has not been proposed for blue laser diodes yet.
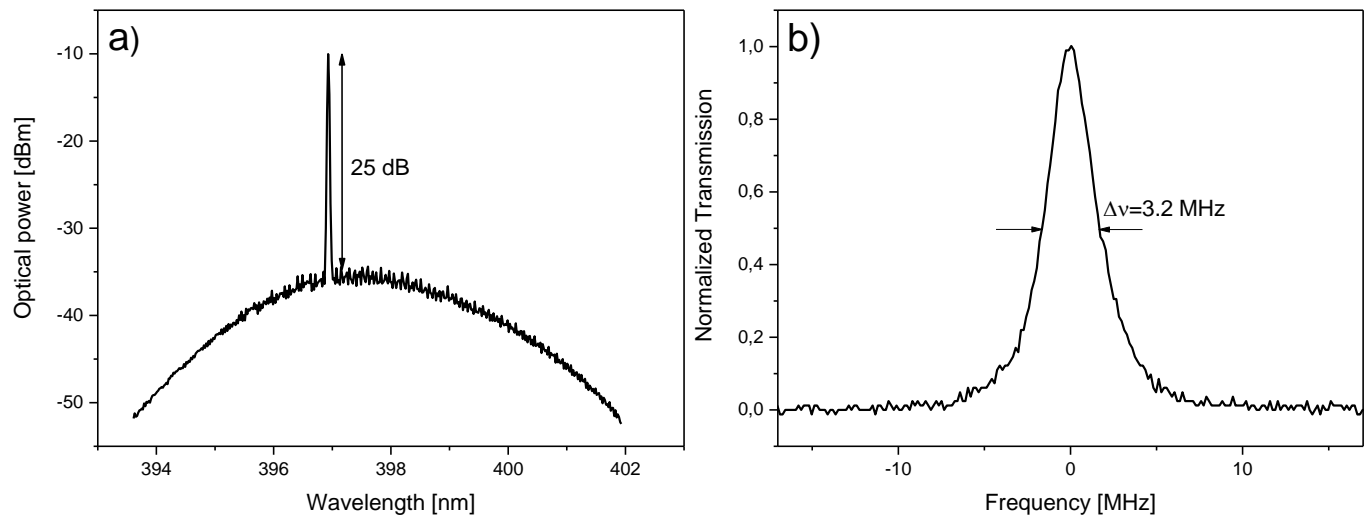

Fig. 1 a) Optical spectrum of the Fiber Bragg Grating external cavity Laser diode (FGL) centered at 396.9 nm b) Resolution limited measurement of the FGL in the single frequency regime measured with a Fabry-Perot Analyser.

In this paper, we demonstrate the possibility to reach single mode emission from a Fabry Perot (FP) InGaN laser diode [5], emitting around $400 \mathrm{~nm}$, by optical feedback using a Fiber Bragg Grating (FBG). Anti reflective coating $(R \approx 2 \%)$ is deposited on the output facet of the FP laser diode to operate the device close from the Reflective Semiconductor Optical Amplifier (RSOA) regime. Then, the output of the laser is coupled to the FBG to be spectrally filtered. A phase-mask based Talbot interferometer arrangement is used to side write the FBG in the photosensitive fiber, single mode at the operating wavelength. It has a uniform profile with $60 \%$ reflectivity centered at $396.9 \mathrm{~nm}$ with a $35 \mathrm{pm}$ bandwidth sufficiently narrow to select one mode of the laser cavity.

Single frequency operation with $25 \mathrm{~dB}$ side mode suppression ratio and a linewidth of less than $3.2 \mathrm{MHz}$ is demonstrated. Such a narrow linewidth laser diode in the blue domain with a compact design could find applications whenever coherency and interferometric resolutions are needed as for laser cooling in optical clock, submarine coherent optical communication or holographic memories.

\section{References}

[1] S. Nakamura, M. Senoh, S. Nagahama, N. Iwasa, T. Yamada, T. Matsushita, H. Kiyoku, and Y. Sugimoto, "InGaN-Based Multi-QuantumWell-Structure Laser Diodes", Jpn. J. Appl. Phys., 35, pp. L74-L76, (1996).

[2] Thomas J. Slight, Szymon Stanczyk, Scott Watson, Amit Yadav, Szymon Grzanka, Edik Rafailov, Piotr Perlin, Stephen P. Najda, Mike Leszczyński, Steffan Gwyn, and Anthony E. Kelly, "Continuous-wave operation of (Al,In)GaN distributed-feedback laser diodes with highorder notched gratings",Applied Physics Express 11, 112701 (2018)

[3] Jun-Ichi Hashimoto, T. Takagi, T. Kato, G. Sasaki, M. Shigehara, K. Murashima, M. Shiozaki, and T. Iwashima, "Fiber-Bragg-Grating External Cavity Semiconductor Laser (FGL) Module for DWDM Transmission", Journal of Lightwave Technology, 21, pp. 2002-2009 (2003) [4] A. Naumenko, P. Besnard, N. Loiko, G. Ughetto and J.C. Bertreux "Characteristics of a semiconductor laser coupled with a fiber Bragg grating with arbitrary amount of feedback", IEEE J. of Quantum Electronics, 39, pp. 1216-1228 (2003)

[5] Antoine Congar, Kamal Hussain, Christelle Pareige, Raphaäel Butté, Nicolas Grandjean, Pascal Besnard and Stéphane Trebaol, "Impact of mode-hopping noise on InGaN edge emitting laser relative intensity noise properties", IEEE J. Quant. Electron. 9197, pp. 1-7 (2017). 